\title{
THE ANALYSIS OF INTER-VARIETAL CHROMOSOME SUB- STITUTIONS IN WHEAT AND THEIR FIRST GENERATION HYBRIDS
}

\author{
C. N. LAW \\ Plant Breeding Institute, Cambridge
}

Received 7.vi.71

\section{INTRODUCTION}

THE ability of the hexaploid wheat, Triticum aestivum, to tolerate the loss of a chromosome allows the substitution of single pairs of homologous chromosomes from one variety into another (Sears, 1953). This provides an opportunity for carrying-out the genetic analysis of this economically useful species with an accuracy not possible using other methods. Comparisons between substitution lines enable the chromosomes responsible for particular varietal differences to be determined. These chromosome assays are however limited in their description of the genetical situation, particularly in the case of quantitative characters, because they make no allowances for the interactions that can occur between chromosomes.

To describe such relationships in meaningful terms requires the application of biometrical procedures. This paper is an account of some of the methods which can be applied to inter-varietal chromosome substitutions and their first generation hybrids.

\section{THEORY}

\section{(a) Detection and description of genetic effects}

Some of the methods by which quantitative differences between substitution lines can be described have been given previously (Law, 1966, 1968; Aksel, 1967). In essence, the methods involve the study of the products of two particular kinds of diallel cross which are conveniently described as Triparental cross 1 and Triparental cross 2 (Aksel, 1967).

Triparental cross 1 involves the hybridisation, in all combinations, of the recipient variety and two substitution lines, whereas cross 2 employs the donor and recipient varieties and a single substitution line as parents.

Both kinds of crosses give rise to a number of comparisons which under certain circumstances enable the detection of additive chromosome effects and two types of departure from additivity, the within- and betweenchromosome interaction effects. In order to describe the procedures involved in the detection of such effects it is best to consider a simplified situation in which only two homologous chromosome pairs are involved, $A-a$ and $B-b$. Suppose also that the donor and recipient varieties have chromosomes which are associated so that their constitutions are $\mathrm{AABB}$ and aabb respectively.

The genotypes produced from the Triparental cross 1 and cross 2 can now be described and are given in table 1. The phenotypic differences among these genotypes are described in terms of the notation of Hayman and Mather (1955), in which $d_{a}$ and $d_{b}$ stand for the difference between the homozygous 
chromosomes, AA-aa and BB-bb; $h_{a}$ and $h_{b}$ represent the effects of dominance and/or non-allelic interaction within a chromosome; $i_{a b}$ refers to the interaction between $d_{a}$ and $d_{b} ; j_{a / b}$ and $j_{b / a}$ distinguish the interaction between $d_{a}$ and $h_{b}$ and $d_{b}$ and $h_{a}$ respectively; and $l_{a b}$ represents the interaction between $h_{a}$ and $h_{b}$.

All these parameters are defined from a common reference, $m$, which in this situation represents the mean of all the possible inbred lines deriving from a cross and is referred to as the F $\infty$ metric (Van der Veen, 1959).

TABLE 1

Triparental crosses. Only the Triparental cross 2 for chromosome $A$ and not chromosome $B$ is shown

Triparental Cross 1

\begin{tabular}{|c|c|c|c|}
\hline & $\mathrm{AAbb}$ & $\mathrm{aaBB}$ & aabb \\
\hline aabb & $\left\{\begin{array}{l}\mathrm{Aabb} \\
m+h_{a}-d_{b}-j_{b} / a\end{array}\right.$ & $\begin{array}{l}\mathrm{aaBb} \\
m-d_{a}+h_{b}-j_{a} / b\end{array}$ & $\begin{array}{l}\text { aabb } \\
m-d_{a}-d_{b}+i_{a b} b\end{array}$ \\
\hline $\mathrm{aaBB}$ & $\left\{\begin{array}{l}\mathrm{AaBb} \\
m+h_{a}+h_{b}+l_{a b}\end{array}\right.$ & $\begin{array}{l}\mathrm{aaBB} \\
m-d_{a}+d_{b}-i_{a b}\end{array}$ & \\
\hline AAbb & $\left\{\begin{array}{l}\mathrm{AAbb} \\
m+d_{a}-d_{b}-i_{a b}\end{array}\right.$ & & \\
\hline
\end{tabular}

Triparental Cross 2 (Chromosome A)

\begin{tabular}{|c|c|c|c|}
\hline & $\mathrm{AABB}$ & $\mathrm{AAbb}$ & aabb \\
\hline aabb & $\left\{\begin{array}{l}\mathrm{AaBb} \\
m+h_{a}+h_{b}+l_{a b}\end{array}\right.$ & $\begin{array}{l}\mathrm{Aabb} \\
m+h_{a}-d_{b}-j_{b} / a\end{array}$ & $\begin{array}{l}\text { aabb } \\
m-d_{a}-d_{b}+i_{a b}\end{array}$ \\
\hline AAbb & $\left\{\begin{array}{l}\mathrm{AABb} \\
m+d_{a}+h_{b}+j_{a} / b\end{array}\right.$ & $\begin{array}{l}\mathrm{AAbb} \\
m+d_{a}-d_{b}-i_{a b}\end{array}$ & \\
\hline $\mathrm{AABB}$ & $\left\{\begin{array}{l}\mathrm{AABB} \\
m+d_{a}+d_{b}+i_{a b}\end{array}\right.$ & & \\
\hline
\end{tabular}

Two types of comparison, detecting additive and within-chromosome interaction effects, can be made among the genotypes derived from each type of cross.

(i) Additive chromosomal effects

Estimate

(a) $\frac{1}{2}(\mathrm{AAbb}-\mathrm{aabb})$

(b) $\frac{1}{2}(\mathrm{aaBB}-\mathrm{aabb})$

$d_{a}-i_{a b}$

$d_{b}-i_{a b}$

(ii) Within-chromosomal interaction effects
(a) $\frac{1}{2}(-\mathrm{AAbb}+2 \mathrm{Aabb}-\mathrm{aabb})$
(b) $\frac{1}{2}(-\mathrm{aaBB}+2 \mathrm{aaBb}-\mathrm{aabb})$
$h_{a}-j_{b / a}$
$h_{b}-j a / b$

All these comparisons give rise to estimates involving between-chromosome interaction parameters. It is thus only when between-chromosome interaction effects are absent that these comparisons provide meaningful estimates of the additive and within-chromosome interaction parameters.

The third informative comparison, detecting between-chromosome interaction, is however different in the two types of cross.

(i) Triparental cross 1

Estimate

$\mathrm{AaBb}-\mathrm{Aabb}-\mathrm{aaBb}+\mathrm{aabb}$

$i_{a b}+j_{a / b}+j_{b / a}+l_{a b}$ 
(ii) Triparental cross 2

(a) Chromosome A

$\mathrm{AABb}-\mathrm{AaBb}-\mathrm{AAbb}+\mathrm{Aabb}$

(b) Chromosome B

$\mathrm{AaBB}-\mathrm{AaBb}-\mathrm{aaBB}+\mathrm{aaBb}$

$$
\begin{aligned}
& i_{a b}+j_{a / b}-j_{b / a}-l_{a b} \\
& i_{a b}-j_{a / b}+j_{b / a}-l_{a b}
\end{aligned}
$$

All of the between-chromosome interaction comparisons provide estimates compounded of four parameters, each of which can take sign so that it is possible to have zero estimates even though the parameters themselves are far from negligible. Furthermore the consequences of this balancing effect depend upon the particular comparisons made. Under some circumstances, only the Triparental cross 1 comparison will detect interactions whereas in other situations interactions will be detected for one of the Triparental cross 2 comparisons only. Also, some circumstances could arise in which none of the Triparental cross comparisons would detect interaction. The complications introduced as a result of balancing are therefore of considerable importance and it is essential to recognise the situations in which failures of detection are likely to arise.

The signs of the four parameters describing between-chromosome interaction among the genotypes which have been specified are determined by the relationships that exist between the donor and recipient chromosomes. These may be associated as in the example used, or they may be dispersed, in which case the signs of the two $j$ 's and $i_{a b}$ will change, but $l_{a b}$ will remain the same. The signs of the $j$ 's and $i_{a b}$ will also change depending on whether the donor or the recipient variety carries the positive or negative acting allele. The effects of these changes in chromosome relationship on the betweenchromosome interaction comparison for both Triparental crosses are summarised in table 2.

\section{TABLE 2}

The effect of chromosome phase and the type of Triparental cross on the signs of the four between-

\begin{tabular}{|c|c|c|c|c|}
\hline \multicolumn{2}{|c|}{ Chromosomes } & Triparental 1 & $\begin{array}{c}\text { Triparental } 2 \\
\text { (Chromosome A) }\end{array}$ & $\begin{array}{c}\text { Triparental } 2 \\
\text { (Chromosome B) }\end{array}$ \\
\hline ssociated & $\begin{array}{l}\text { Donor AABB } \\
\text { Donor aabb }\end{array}$ & $\begin{array}{l}i_{a b}+j_{a / b}+j_{b / a}+l_{a b} \\
i_{a b}-j_{a / b}-j_{b / a}+l_{a b}\end{array}$ & $\begin{array}{l}i_{a b}+j_{a / b}-j_{b / a}-l_{a b} \\
i_{a b}-j_{a / b}+j_{b / a}-l_{a b}\end{array}$ & $\begin{array}{l}i_{a b}-j_{a / b}+j_{b / a}-l_{a b} \\
i_{a b}+j_{a / b}-j_{b / a}-l_{a b}\end{array}$ \\
\hline isperse & $\begin{array}{l}\text { Donor AAbb } \\
\text { Donor aaBB }\end{array}$ & $\begin{array}{l}-i_{a b}+j_{a / b}-j_{b / a}+l_{a b} \\
-i_{a b}-j_{a / b}+j_{b / a}+l_{a b}\end{array}$ & $\begin{array}{l}-i_{a b}+j_{a / b}+j_{b / a}-l_{a b} \\
-i_{a b}-j_{a / b}-j_{b / a}-l_{a b}\end{array}$ & $\begin{array}{l}-i_{a b}-j_{a / b}-j_{b / a}-l_{a b} \\
-i_{a b}+j_{a / b}+j_{b / a}-l_{a b}\end{array}$ \\
\hline
\end{tabular}
chromosome interaction parameters

The parameters $i_{a b}, j_{a / b}, j_{b / a}$ and $l_{a b}$ also have particular relationships depending on the nature of the interaction. For the two major types of classical gene interaction, complementary and duplicate action, the signs of the four parameters behave in a characteristic manner. When $h$ is positive, $i_{a b}, j_{a / b}, j_{b / a}$, and $l_{a b}$ are positive for complementary gene action and negative for duplicate gene action. When $h$ is negative then for complementary interaction $i_{a b}$ and $l_{a b}$ are also negative whereas the two $j$ 's are positive. Duplicate interactions, on the other hand, have $i_{a b}$ and $l_{a b}$ positive and the $j$ 's negative and correlated with $h$ (Mather, 1967).

The effects of these variations on the ability to detect between-chromosome interaction can be explored by supposing that $i_{a b}=j_{a / b}=j_{b / a}=l_{a b}$ and substituting values of 1 or -1 , according to the type of interaction, for 
the parameters given in table 2. The results of this exercise are presented in table 3 .

It is apparent that for many comparisons a balance occurs between the parameters so that it is likely that interactions will be difficult to detect in these cases. For those comparisons in which the parameters reinforce each other to give estimates of 4 or -4 , large differences exist between the two Triparental crosses. When the chromosomes are associated, estimates indicating interaction occur only for some of the comparisons carried out for Triparental cross 1 . On the other hand, when the chromosomes are dispersed, the reverse situation occurs and it is Triparental cross 2 which is the more efficient at detecting interaction. In half of the associated cases, however, neither of the Triparental crosses provide comparisons in which the parameters reinforce each other.

TABLE 3

The ability to detect between-chromosome interactions for each of the Triparental crosses when $\mathrm{i}_{\mathrm{ab}}=\mathrm{j}_{\mathrm{a} / \mathrm{b}}$ $=\mathrm{j}_{\mathrm{b} / \mathrm{a}}=l_{\mathrm{ab}}=1$ or -1

Chromosomes Type of interaction Triparental $1 \quad$ Triparental 2 Triparental 2

Associated

\begin{tabular}{|c|c|c|c|c|c|}
\hline \multirow[t]{4}{*}{ Donor AABB } & Complementary & $h+v e$ & 4 & 0 & 0 \\
\hline & Duplicate & $\mathrm{h}+\mathrm{ve}$ & -4 & 0 & 0 \\
\hline & Complementary & $h-v e$ & 0 & 0 & 0 \\
\hline & Duplicate & $\mathrm{h}$-ve & 0 & 0 & 0 \\
\hline \multirow[t]{4}{*}{ Donor aabb } & Complementary & $h+v e$ & 0 & 0 & 0 \\
\hline & Duplicate & $\mathrm{h}+\mathrm{ve}$ & 0 & 0 & 0 \\
\hline & Complementary & $\mathrm{h}-\mathrm{ve}$ & -4 & 0 & 0 \\
\hline & Duplicate & $\mathrm{h}-\mathrm{ve}$ & 4 & 0 & 0 \\
\hline \multicolumn{6}{|c|}{ Dispersed } \\
\hline \multirow[t]{4}{*}{ Donor AAbb } & Complementary & $h+v e$ & 0 & 0 & -4 \\
\hline & Duplicate & $\mathrm{h}+\mathrm{ve}$ & 0 & 0 & 4 \\
\hline & Complementary & $\mathrm{h}-\mathrm{ve}$ & 0 & 4 & 0 \\
\hline & Duplicate & $\mathrm{h}-\mathrm{ve}$ & 0 & -4 & 0 \\
\hline \multirow[t]{4}{*}{ Donor aaBB } & Complementary & $h+v e$ & 0 & -4 & 0 \\
\hline & Duplicate & $\mathrm{h}+\mathrm{ve}$ & 0 & 4 & 0 \\
\hline & Complementary & $h-v e$ & 0 & 0 & 4 \\
\hline & Duplicate & $\mathrm{h}-\mathrm{ve}$ & 0 & 0 & \\
\hline
\end{tabular}

The detection of interaction where balanced effects occur may be possible if $i_{a b} \neq j_{a / b} \neq j_{b / a} \neq l_{a b}$ and its efficiency will depend upon whether the $j$ 's combined are greater or smaller than $i_{a b}$ and $l_{a b}$. It is possible therefore if both Triparental crosses are made that this situation will arise in one or other of the crosses, so that the presence of interaction will be detected. In general, however, it might be expected that the magnitude of the summed interactions in these instances will be so low as to make detection difficult.

The results presented in table 3 clearly point to the need for carrying out both types of Triparental cross. This means in fact the production of a Quadruple cross which is of course the combination of both the Triparental crosses and involves intercrossing the donor and recipient varieties, and two substitution lines.

When this is carried out it is possible to detect most of the situations in which between-chromosome interaction may occur and furthermore to determine whether the interaction is of a complementary or duplicate nature. It will also enable the recognition of those situations where detection may be 
difficult, because estimates of $d$ and $h$ for each chromosome will be obtainable and so will allow a complete description of the relationships between the chromosomes involved. It would therefore be possible to introduce a degree of caution into extrapolating from such situations.

An important restriction which should be mentioned applies to some of the comparisons based on Triparental cross 2 . Both the donor and recipient varieties are used in this type of cross so that the differences among the resultant genotypes involve not only the substituted chromosomes, as is the case in the simplified model used here, but also the remaining chromosomes of the wheat background. The between-chromosome interaction comparisons therefore can include parameters which relate to interactions involving chromosomes other than A-a and B-b. To detect interactions for the chromosome A comparison, for example, could consequently indicate that this chromosome interacts with chromosome $\mathrm{B}$ or that it interacts with an unknown chromosome in the background.

\section{(b) Estimation by the method of least squares}

The comparisons which have been described so far are similar to the "scaling" tests used by Mather (1949). Such tests are concerned mainly with detection. Although estimates of the parameters can be achieved by this method, the resulting estimates often have such large standard errors that meaningful interpretation may be difficult. Also it is impossible to achieve estimates of some of the parameters for the comparisons given here and where estimates can be made, for example $d$ and $h$, it is necessary to show that between-chromosome interactions are absent.

The method of least squares has been used by Mather (1949), Cavalli, (1952), Jinks (1956), and Jinks and Perkins (1969) to estimate genetic parameters and overcomes many of these difficulties. This procedure involves the specificaion of a genetic model using the parameters defined earlier in this paper, the estimation of the proposed parameters using least squares, the derivation of expectations based upon these estimates, and the determination of "goodness of fit" by calculating the observed-expected deviations. The method therefore not only provides the means whereby more precise estimates can be achieved, but also, since it is possible to go through several " fitting" processes, to test for the presence or absence of certain major genetic effects. For example, considering the crosses described previously, an additive model could first be attempted, so that the "fitting " process would effectively provide a test for the presence or absence of non-additive effects. Likewise a model consisting of additive and within-chromosome interaction effects would provide a test for between-chromosome interaction and so on.

In general it appears that the least squares method will detect betweenchromosome interactions for exactly those situations which are detectable using the straight comparisons. When the assumption is made that all the between-chromosome interaction parameters are equal, then comparisons between the two approaches indicate that both are equally effective. Whether the least squares method will be more efficient at detecting interaction when this assumption does not apply is not certain.

Since the limit to the number of parameters which may be estimated is determined by the number of observations, the Quadruple cross will provide greater opportunities for estimation than either of the Triparental crosses in 
which fewer observations are of course involved. The analysis of the Quadruple cross is based upon ten genotypes, so that a maximum of nine parameters may be estimated and their adequacy tested. Five of these will be the parameters already used in describing the differences between the two chromosomes, A-a and B-b, these are $d_{a}, d_{b}, h_{a}, h_{b}$ and $m$. Two parameters, $d_{c}$ and $h_{c}$, will also be required to make allowance for the remaining chromosomes of the wheat complement. This then leaves two parameters which can be estimated to describe the between-chromosome interactions. These may be allocated in a number of ways but for the present the parameters will be restricted to describing the interactions between the substituted chromosomes, A-a and B-b.

Four parameters have so far been used to describe the interactions between these chromosomes, so that it is necessary to introduce a simplifying assumption which reduces the number of parameters to two. It has been shown that the distinction between complementary and duplicate interactions can be described in terms of the signs of the four parameters. In every instance, however, $i$ and $l$ are correlated as indeed are the two $j$ 's. This therefore indicates that the most reasonable simplifying assumption that can be made and one that is likely to impair the efficiency of estimation the least is to state that $i_{a b}=l_{a b}$ and $j_{a / b}=j_{b / a}$ and so reduce the number of between-chromosome interaction parameters to two. For the present purposes these two parameters may be donated as $r_{a b}$ and $s_{a b}$, where $r_{a b}=i_{a b}=l_{a b}$ and $s_{a b}=j_{a / b}=j_{b / a}$.

If this model proves to be inadequate then either $i_{a b} \neq l_{a b}$ or $j_{a / b} \neq j_{b / a}$, or interactions involving chromosomes of the background are concerned.

\section{EXPERIMENT}

In order to illustrate the methods which have been described, a Quadruple cross was carried out involving the varieties Hope and Chinese Spring (CS) and two of the substitution lines of Hope into GS, CS (Hope 5A) and CS (Hope 5D), in which chromosomes 5A and 5D of Hope have replaced separately their homologues in CS.

Both these chromosomes belong to the same homoeologous group (Sears, 1953) so that their gene action can be presumed to be very similar. Under such circumstances, interactions of a duplicate nature would reasonably be expected to occur, since in essence the chromosomes of a homoeologous group provide a naturally occurring dosage series of identical loci.

The plants obtained from the Quadruple cross were grown under controlled environment conditions of continuous light and at a temperature of $18 \pm 1^{\circ} \mathrm{C}$. Plants were grown singly in pots and a randomised block layout consisting of six replications used. Each block was composed of 20 pots, made up of the six hybrid genotypes and their reciprocals, and duplicated parental lines. The character studied was the days to ear-emergence.

\section{Results}

The mean ear-emergence times for each genotype summing over reciprocals and duplicates are given in table 4 . The analysis of this data is shown in table 5 and indicates the highly significant genotypic effects and the complete absence of reciprocal differences. Also, the variances for each of the genotypic means are homogeneous (Bartlett Test, $\left.\chi_{(9)}^{2}\right)=12 \cdot 50, P=0 \cdot 2-0 \cdot 1$ ), 
TABLE 4

Mean ear-emergence times for the ten genotypes of the Quadruple cross. A-a, describes the two homologues of chromosomes $5 A ; B-b$, chromosome $5 D$; and C-c the remaining chromosomes of the background

$\begin{array}{lllll} & \begin{array}{l}\text { Hope } \\ \text { aaBBcc }\end{array} & \begin{array}{c}\text { CS } \\ \text { AAbbCC }\end{array} & \begin{array}{c}\text { CS/Hope 5A } \\ \text { aabbCC }\end{array} & \begin{array}{r}\text { CS/Hope 5D } \\ \text { AABBCC }\end{array} \\ \text { CS/Hope 5D } & \text { AaBBCc } & \text { AABbCC } & \text { AaBbCC } & \text { AABBCC } \\ \text { AABBCC } & 45.0000 & 56.1667 & 49.7500 & 91.9167 \\ \text { CS/Hope 5A } & \text { aaBbCc } & \text { AabbCC } & \text { aabbCC } & \\ \text { aabbCC } & 41.830 & 49.5000 & 41.9167 & \\ \text { CS } & \text { AaBbCc } & \text { AAbbCC } & & \\ \text { AAbbCC } & 46.6667 & 56.5833 & & \\ \text { Hope } & \text { aaBBcc } & & & \\ \text { aaBBcc } & 44.3333 & & & \end{array}$

so that the standard errors used either for making comparisons or for determining the significance of a particular estimate have been based upon the pooled reciprocal and error items.

The estimates of the various genetic effects using the straight comparisons described in the previous section can now be obtained. The additive effects for each of the chromosomes are $d_{5 \mathrm{~A}}=-7.33 \pm 0.51$ and $d_{5 \mathrm{D}}=17.67 \pm 0.51$. Likewise the within-chromosome interaction estimates turn-out to be $h_{5 \mathrm{~A}}$ $=0.25 \pm 0.89$ and $h_{5} \mathrm{D}=17 \cdot 17 \pm 0.89$. Apart from $h_{5 \mathrm{~A}}$, all these estimates are highly significant $(P<0.001)$.

The more important comparisons however concern the detection of between-chromosome interaction since, using this model, it is only in the absence of such effects that the detection and estimation of the additive and the within-chromosome interaction effects are meaningful.

\begin{tabular}{|c|c|c|c|c|}
\hline \multicolumn{5}{|c|}{ TABLE 5} \\
\hline Item & d.f. & M.S. & V.R. & $\mathbf{P}$ \\
\hline Blocks & $\begin{array}{l}5 \\
0\end{array}$ & $\begin{array}{r}25.8680 \\
2636.0633\end{array}$ & & \\
\hline $\begin{array}{l}\text { Genotypes } \\
\text { Duplicates + } \\
\text { Reciprocals }\end{array}$ & $\begin{array}{r}9 \\
10\end{array}$ & $\begin{array}{r}2636.9633 \\
6.2420\end{array}$ & 418.8049 & $<0.001$ \\
\hline Error & 95 & $6 \cdot 2964$ & & \\
\hline
\end{tabular}

The relevant comparisons are best illustrated if the chromosomes $5 \mathrm{~A}$ and $5 \mathrm{D}$ are described using the symbols $\mathrm{A}-\mathrm{a}$ and $\mathrm{B}-\mathrm{b}$ respectively. In this instance, however, and unlike the illustration previously given, the donor and recipient varieties have chromosomes which are dispersed. This follows from the fact that CS (Hope 5A) is earlier than CS, whereas CS (Hope 5D) is much later. Also the background chromosome differences can be described using the symbols $\mathrm{C}-\mathrm{c}$, where $\mathrm{CG}$ refers to the chromosomes from Ghinese Spring and cc to those from Hope. The four genotypes used in the cross can therefore be depicted as aaBBcc and AAbbCG for the donor and recipient varieties, and aabbCC and $A A B B C G$ for the two substitution lines, CS (Hope 5A) and CS (Hope 5D) respectively.

The between-chromosome interaction comparison for the Triparental cross 1 can now be given and is composed of the following genotypes:

$$
\begin{aligned}
& \text { AaBbCC }- \text { AABbCC }-A a b b C C+A A b b C G \\
= & (49.750-56 \cdot 167-49.500+56.583) \\
= & +0.666 \pm 1.448 .
\end{aligned}
$$


There is consequently no evidence of between-chromosome interaction using this test. Turning to the Triparental cross 2 comparisons, the genotypes used to detect interactions involving chromosome $5 \mathrm{~A}$ or $\mathrm{A}-\mathrm{a}$ using the present nomenclature are:

$$
\begin{aligned}
& a a B b C c-A a B b C c-a a b b C C+A a b b C C \\
= & (41.830-46.667-41.917-+49.500) \\
= & +2 \cdot 746 \pm 1.448 .
\end{aligned}
$$

Again this comparison provides no evidence of between-chromosome interactions. There remains the comparison involving chromosome $5 \mathrm{D}$ or $\mathrm{B}-\mathrm{b}$, which is :

$$
\begin{aligned}
& \mathrm{AaBBCc}-\mathrm{AaBbCc}-\mathrm{AABBCC}+\mathrm{AABbCC} \\
= & (45 \cdot 000-46 \cdot 667-91 \cdot 917+56 \cdot 167) \\
= & -37 \cdot 417 \pm 1 \cdot 448 .
\end{aligned}
$$

This negative estimate is highly significant $(\mathbf{P}<0 \cdot 001)$ so that chromosome $5 \mathrm{D}$ interacts with another chromosome or chromosomes of the complement.

The information obtained from these comparisons can now be collated with the variable interaction situations explored previously and summarised in table 3. In the present experiment, the donor parent, Hope, has chromosomes $5 \mathrm{~A}$ and $5 \mathrm{D}$ in the dispersed condition, i.e. aaBB, the within-chromosome interaction estimates appear to be negative and chromosome B-b (5D) and not chromosome $\mathrm{A}-\mathrm{a}(5 \mathrm{~A})$ gives a negative between-chromosome interaction estimate. These results exactly fit the last situation given in table 3 in which duplicate interactions occur between-chromosome A-a and B-b. This is of course the kind of interaction expected when the chromosomes under study belong to the same homoeologous group and consequently provides support for the hypothesis that chromosome $5 \mathrm{~A}$ is involved in the interaction with chromosome 5D and not some other chromosome of the complement.

The results can now be considered using the method of least squares. As a first step, a genetic model is proposed in which only the additive and withinchromosome interaction effects are involved. For the Quadruple cross this means estimating the seven parameters already defined in Section $2, m$, $d_{a}, d_{b}, d_{c}, h_{a}, h_{b}$ and $h_{c}$.

The estimates obtained for these parameters are shown in table 6 . Expectations derived from these estimates enable deviations from the observed values to be calculated and a test of the model's adequacy to be carried out. The sum of the squared deviations turns out to be $400 \cdot 6478$ and will have three degrees of freedom since out of a total of ten degrees of freedom, seven have been used in fitting the parameters. The mean square deviation is therefore $400.6478 / 3=133.5393$ which can be compared with an error variance of $6.2912 / 12=0.5243$ since the means used in estimating the parameters are based on twelve observations. This gives rise to a V.R. $=254.47$ which is significant $(\mathrm{P}<0.001)$. The additive and withinchromosome interaction model is therefore not capable of explaining the data and between-chromosome interactions must be proposed.

The two parameters $r_{a b}$ and $s_{a b}$, defining interactions between chromosomes $5 \mathrm{~A}$ and $5 \mathrm{D}$ can now be added to the model to give nine parameters altogether. The estimates of these parameters are also given in table 6 . Expectations derived from these estimates give almost a perfect fit with the observations. The sum of squares of deviations in this case is 0.1112 which 
since nine parameters have been estimated will have one degree of freedom. This is smaller than the mean square error variation of 0.5243 so that the proposed genetic model allowing for between-chromosome interaction gives a close agreement with the observed values.

The signs of the estimates indicate the kind of interaction occurring between the chromosomes. In the present example, all the within-chromosome interaction estimates are significant and are negative, also both the between-chromosome interaction estimates are significant but in this case $r_{a b}$ is positive and $s_{a b}$ negative. These relationships are of course those found when duplicate interactions occur (Mather, 1967).

The two kinds of analysis therefore agree. Duplicate interactions occurring between the related chromosomes $5 \mathrm{~A}$ and $5 \mathrm{D}$ could account for the observed variation.

TABLE 6

Estimates of the genetic parameters

$\begin{array}{ccc}\text { Estimate } & \begin{array}{c}\text { Additive and within- } \\ \text { chromosome interaction } \\ \text { model }\end{array} & \begin{array}{c}\text { Additive within- and } \\ \text { between-chromosome } \\ \text { interaction model }\end{array} \\ m & 51.3253 \pm 0.4751^{* * *} & 58.8959 \pm 0.6773^{* * *} \\ d_{a} & 6.7246 \pm 0.4180^{* * *} & 15.7708 \pm 0.5724^{* * *} \\ d_{b} & 11.5386 \pm 0.4180^{* * *} & 9.2292 \pm 0.5724^{* * *} \\ d_{c} & 11.8090 \pm 0.6329^{* * *} & -0.5000 \pm 1.0079 \\ h_{a} & -6.7611 \pm 0.5120^{* * *} & -9.6042 \pm 0.5431^{* * *} \\ h_{b} & -4.8861 \pm 0.5120^{* * *} & -7.7292 \pm 0.5431^{* * *} \\ h_{c} & -0.5736 \pm 0.6773 & -3.4167 \pm 0.7011^{* * *} \\ r_{a b} & - & 8.5209 \pm 0.5431^{* * *} \\ s_{a b} & - & -10.1042 \pm 0.4434^{* * *} \\ & & \end{array}$

\section{Conclusion}

The conclusions which can be drawn from the outline of the theory and its application to a particular experimental situation can be readily made.

If analysis involving inter-varietal chromosome substitutions is restricted to the observations made on the lines themselves and their first generation hybrids, then the Quadruple cross in which both the donor and recipient varieties and two substitution lines are used as parents is the most informative. Neither of the Triparental crosses used in previous analyses (Law, 1966, 1968; Aksel, 1967) is capable of detecting between-chromosome interactions under all genetic conditions and situations. Although a similar criticism can be levelled at the Quadruple cross, the failure to recognise these departures from additivity is not as extensive.

So far as estimation is concerned neither of the Triparental crosses provide sufficient observations to allow the complete separation of additive, withinchromosome and between-chromosome interaction effects. The Quadruple cross, on the other hand, will enable the estimation of certain betweenchromosome interactions. Under some circumstances, therefore, Triparental crosses may detect between-chromosome interactions but it will not be possible to estimate any useful parameters which could be used to specify and measure the magnitude of such interactions. The ability to predict the phenotypes of other genetic situations which could arise by manipulating the genetic variation under study will consequently be reduced. 
Having made these points in favour of the Quadruple cross situation, it is necessary to emphasise again the incomplete genetic description which can arise, given certain conditions when this type of cross is used. One quarter of the between-chromosome interaction situations described previously suffered from the consequences of balanced genetic effects which could not be overcome by extending the method to the Quadruple cross. Likewise it has been mentioned that although between-chromosome interactions have been detected, and estimates made of their effects in such a way as to give a close fit with the observed values, it is still not definitive proof that the model proposed is correct. Other models could also be produced which are capable of explaining the data. This position is very apparent when the contributions of the background chromosomes are considered in the example described. Although the analysis has shown that the two chromosomes, $5 \mathrm{~A}$ and $5 \mathrm{D}$, could interact in a duplicate manner, the results of the Triparental cross 2 comparisons indicate that it is only chromosome $5 \mathrm{D}$ which can be definitely implicated in the interaction. Chromosomes other than 5A could therefore be involved and it is impossible using the analyses described earlier, including the analysis by means of least squares, to determine whether this is so or not. The analyses have therefore proved that chromosome $5 \mathrm{D}$ interacts in a duplicate manner with another chromosome of the wheat complement and that the results are consistent with this chromosome being $5 \mathrm{~A}$.

There are of course $a$ priori grounds for considering that chromosomes $5 \mathrm{~A}$ and $5 \mathrm{D}$ in wheat are involved in a duplicate interaction, so that the close agreement of observation with this hypothesis provides strong grounds for its acceptance. On the other hand, the final proof can only be obtained as a result of further experiment, in which the information obtained from further generations is necessary.

The example chosen must therefore be considered as providing an illustration of a method applicable to a wide range of genetic situations involving chromosome substitution lines in wheat. The method has disadvantages but these have been accurately delimited so that possible failures can be detected and appropriate allowances made.

\section{Summary}

1. The two kinds of crossing scheme, Triparental 1 and Triparental 2, which have been used previously to study inter-varietal chromosome substitutions in wheat and their first generation hybrids are reconsidered from the point of view of biometry.

2. It is pointed out that the ability to detect additive, within- and between-chromosome interaction effects, using either of the Triparental crosses and using any number of comparative tests among the genotypes derived from these crosses, suffer from the consequences of balanced effects among the sets of parameters involved in making a particular comparison.

3. The effects of chromosomal phase and type of chromosome interaction on the ability to detect between-chromosome interaction are explored. Triparental 1 is the more efficient of the two types of cross at detecting between-chromosome interactions when the chromosomes in the donor and recipient varieties are associated, whereas Triparental 2 is more efficient when the chromosomes are dispersed. In some instances, neither cross is effective in detecting between-chromosome interaction. 
4. In order to overcome this deficiency it is concluded that the best crossing design is a combination of both Triparental 1 and 2 to give what is termed the Quadruple cross.

5. Detection and estimation using least squares procedures are briefly considered and it appears that the ability to detect certain genetic effects is very similar to that obtained using comparative tests.

6. These different types of analysis are applied to the results obtained from a Quadruple cross in which the varieties Hope and Chinese Spring (CS) along with two substitutions lines CS (Hope 5A) and CS (Hope 5D) are used as parents. The analysis indicates that for the character, days to ear-emergence, chromosome $5 \mathrm{D}$ interacts in a duplicate manner with another chromosome of the wheat complement and the results are consistent with this being the related chromosome $5 \mathrm{~A}$.

\section{REFERENCES}

AKSELL, R. 1967. Quantitative genetic analysis of characters in wheat using chromosome substitution lines (Theoretical considerations). Genetics, 57, 195-211.

GAVALLI, L. L. 1952. An analysis of linkage in quantitative inheritance. Quantitative Inheritance (Eds. E. C. Reeve and C. H. Waddington). H.M.S.O. (London), 135-144. hAYMAN, B. I., AND MATHER, $\mathbf{x}$. 1955. The description of genic interactions in continuous variation. Biometrics, 2, 69-82.

JINKS, J. L., AND PERKINS, JEAN M. 1969. The detection of linked epistatic genes for a metrical trait. Heredity, 24, 465-475.

JNxx, J. L. 1956. The $\mathrm{F}_{2}$ and backcross generations from a diallel set of crosses. Heredity, $10,1-30$.

LAW, C. N. 1966. Biometrical analysis using chromosome substitutions within a species. In Chromosome manipulation in plant genetics (Eds. R. Riley and K. R. Lewis). Suppt. Heredity, 20, 59-85.

LAW, c. N. 1968. Genetic analysis using inter-varietal chromosome substitutions. Proc. 3rd Int. Wheat Genet. Symp., Canberra, 331-342.

MATHER, X. 1949. Biometrical Genetics. Methuen, London.

MATHER, X. 1967. Complementary and duplicate gene interactions in biometrical genetics. Heredity, 22, 97-103.

SEARS, E. R. 1953. Nullisomic analysis in common wheat. Am. Naturalist, 87, 245-252.

VAN DER VEEN, J. H. 1959. Tests of non-allelic interaction and linkage for quantitative characters in generations derived from two diploid pure lines. Genetica, 30, 201-232. 